\title{
A measurement of the average lifetime of b-flavoured baryons
}

\author{
OPAL Collaboration
}

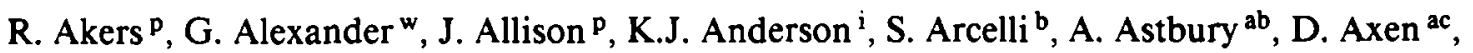
G. Azuelos ${ }^{\text {r,l }}$, J.T.M. Baines ${ }^{\text {p }}$, A.H. Ball ${ }^{q}$, J. Banks $^{\text {p }}$, R.J. Barlow ${ }^{\text {p }}$, S. Barnett ${ }^{\text {p }}$, R. Bartoldus ${ }^{c}$, J.R. Batley ${ }^{\mathrm{e}}$, G. Beaudoin ${ }^{\mathrm{r}}$, A. Beck ${ }^{w}$, G.A. Beck ${ }^{\mathrm{m}}$, J. Becker ${ }^{\mathrm{j}}$, C. Beeston ${ }^{\mathrm{p}}$, T. Behnke ${ }^{\mathrm{aa}}$, K.W. Bell ${ }^{t}$, G. Bella ${ }^{w}$, P. Bentkowski ${ }^{r}$, P. Berlich ${ }^{j}$, S. Bethke ${ }^{k}$, O. Biebel ${ }^{\text {c }}$, I.J. Bloodworth ${ }^{a}$, P. Bock ${ }^{k}$, B. Boden ${ }^{c}$, H.M. Bosch ${ }^{k}$, M. Boutemeur ${ }^{r}$, H. Breuker ${ }^{h, 2}$, P. Bright-Thomas ${ }^{y}$, R.M. Brown ${ }^{\text {i }}$, A. Buijs ${ }^{\text {h }}$, H.J. Burckhart ${ }^{\text {b }}$, C. Burgard ${ }^{a a}$, P. Capiluppi ${ }^{b}$, R.K. Carnegie ${ }^{f}$, A.A. Carter ${ }^{\text {m }}$, J.R. Carter ${ }^{\mathrm{e}}$, C.Y. Chang ${ }^{\text {q }}$, D.G. Charlton ${ }^{\text {h }}$, S.L. Chu ${ }^{\text {d }}$, P.E.L. Clarke ${ }^{\circ}$, J.C. Clayton ${ }^{\text {a }}$, I. Cohen ${ }^{\text {w }}$, J.E. Conboy ${ }^{\circ}$, M. Cooper ${ }^{v}$, M. Coupland ${ }^{n}$, M. Cuffiani ${ }^{b}$, S. Dado ${ }^{v}$, G.M. Dallavalle ${ }^{b}$, S. De Jong ${ }^{m}$, L.A. del Pozo ${ }^{\mathrm{c}}$, H. Deng ${ }^{\mathrm{q}}$, A. Dieckmann ${ }^{\mathrm{k}}$, M. Dittmar $^{\mathrm{d}}$, M.S. Dixit ${ }^{8}$, E. do Couto e Silva ${ }^{\ell}$, J.E. Duboscq ${ }^{\text {h }}$, E. Duchovni ${ }^{2}$, G. Duckeck ${ }^{k}$, I.P. Duerdoth ${ }^{\text {p }}$, D.J.P. Dumas ${ }^{f}$, P.A. Elcombe ${ }^{e}$, P.G. Estabrooks ${ }^{f}$, E. Etzion ${ }^{w}$, H.G. Evans ${ }^{i}$, F. Fabbri ${ }^{b}$, B. Fabbro ${ }^{\text {}}$, M. Fierro ${ }^{b}$, M. Fincke-Keeler ${ }^{\text {ab }}$, H.M. Fischer ${ }^{c}$, D.G. Fong ${ }^{q}$, M. Foucher ${ }^{\mathrm{q}}$, A. Gaidot ${ }^{\text {}, ~ J . W . ~ G a r y ~}{ }^{\mathrm{d}}$, J. Gascon ${ }^{\mathrm{r}}$, N.I. Geddes ${ }^{\mathfrak{t}}$, C. Geich-Gimbel ${ }^{\mathrm{c}}$, S.W. Gensler ${ }^{i}$, F.X. Gentit ${ }^{\text {, }}$, G. Giacomelli ${ }^{b}$, R. Giacomelli ${ }^{b}$, V. Gibson ${ }^{\mathrm{e}}$, W.R. Gibson ${ }^{\mathrm{m}}$, J.D. Gillies ${ }^{t}$, J. Goldberg ${ }^{\text {, }}$, D.M. Gingrich ${ }^{\text {ad, } 1}$, M.J. Goodrick ${ }^{\mathrm{e}}$, W. Gorn ${ }^{\mathrm{d}}$, C. Grandi ${ }^{\mathrm{b}}$, F.C. Grant ${ }^{\mathrm{e}}$, J. Hagemann ${ }^{\mathrm{aa}}$, G.G. Hanson ${ }^{\ell}$, M. Hansroul ${ }^{\mathrm{h}}$, C.K. Hargrove ${ }^{\mathrm{g}}$, P.F. Harrison ${ }^{\mathrm{m}}$, J. Hart ${ }^{\text {h }}$, P.M. Hattersley ${ }^{\text {a }}$, M. Hauschild ${ }^{\text {h }}$, C.M. Hawkes ${ }^{\text {h }}$, E. Heflin ${ }^{\text {d }}$, R.J. Hemingway ${ }^{f}$, G. Herten $^{j}$, R.D. Heuer ${ }^{\text {h }}$, J.C. Hill ${ }^{\text {e }}$, S.J. Hillier ${ }^{\text {h }}$, T. Hilse ${ }^{j}$, D.A. Hinshaw ${ }^{\mathrm{r}}$, J.D. Hobbs ${ }^{\text {, }}$, P.R. Hobson ${ }^{y}$, D. Hochman ${ }^{z}$, R.J. Homer ${ }^{a}$, A.K. Honma ${ }^{\text {ab, }, ~}$, R.E. Hughes-Jones ${ }^{\text {, }}$,

R. Humbert ${ }^{j}$, P. Igo-Kemenes ${ }^{k}$, H. Ihssen ${ }^{k}$, D.C. Imrie ${ }^{y}$, A.C. Janissen ${ }^{f}$, A. Jawahery ${ }^{q}$, P.W. Jeffreys ${ }^{t}$, H. Jeremie ${ }^{r}$, M. Jimack ${ }^{a}$, M. Jones ${ }^{a c}$, R.W.L. Jones ${ }^{h}$, P. Jovanovic ${ }^{a}$, C. Jui ${ }^{d}$, D. Karlen ${ }^{f}$, K. Kawagoe ${ }^{x}$, T. Kawamoto ${ }^{x}$, R.K. Keeler ${ }^{\text {ab }}$, R.G. Kellogg ${ }^{\text {q }}$, B.W. Kennedy ${ }^{\text {, }}$, J. King ${ }^{\mathrm{m}}$, S. Kluth ${ }^{\mathrm{e}}$, T. Kobayashi ${ }^{\mathrm{x}}$, D.S. Koetke ${ }^{\mathrm{h}}$, T.P. Kokott ${ }^{c}$, S. Komamiya ${ }^{x}$, J.F. Kral ${ }^{\text {h }}$, R. Kowalewski ${ }^{h}$, J. von Krogh ${ }^{k}$, J. Kroll ${ }^{i}$, P. Kyberd ${ }^{m}$, G.D. Lafferty ${ }^{p}$, H. Lafoux ${ }^{u}$,

R. Lahmann ${ }^{q}$, J. Lauber ${ }^{\text {h }}$, J.G. Layter ${ }^{d}$, P. Leblanc ${ }^{r}$, A.M. Lee ${ }^{\text {ae }}$, E. Lefebvre ${ }^{r}$, M.H. Lehto ${ }^{\circ}$, D. Lellouch ${ }^{z}$, C. Leroy $^{r}$, J. Letts ${ }^{d}$, L. Levinson ${ }^{z}$, S.L. Lloyd ${ }^{m}$, F.K. Loebinger ${ }^{p}$, J.M. Lorah $^{q}$, B. Lorazo ${ }^{\text {r }}$, M.J. Losty ${ }^{8}$, X.C. Lou ${ }^{\ell}$, J. Ludwig $^{j}$, A. Luig ${ }^{j}$, M. Mannelli ${ }^{h}$, S. Marcellini ${ }^{b}$, C. Markus ${ }^{\text {c }}$, A.J. Martin ${ }^{\mathrm{m}}$, J.P. Martin ${ }^{\mathrm{r}}$, T. Mashimo ${ }^{\mathrm{x}}$, P. Mättig ${ }^{\mathrm{c}}$, U. Maur ${ }^{\mathrm{c}}$, J. McKenna $^{\text {ac }}$, T.J. McMahon ${ }^{\text {a }}$, J.R. McNutt ${ }^{y}$, F. Meijers ${ }^{\text {h }}$, D. Menszner ${ }^{k}$, F.S. Merritt ${ }^{i}$, H. Mes ${ }^{g}$, A. Michelini ${ }^{\text {h }}$, R.P. Middleton ${ }^{\text {' }}$, G. Mikenberg ${ }^{\text {z }}$, J. Mildenberger ${ }^{\mathrm{f}}$, D.J. Miller ${ }^{\mathrm{o}}, \mathbf{R}$. Mir $^{\ell}$, W. Mohr ${ }^{j}$, C. Moisan ${ }^{\mathrm{r}}$, A. Montanari ${ }^{\mathrm{b}}$, T. Mori ${ }^{x}$, M. Morii ${ }^{x}$, U. Müller ${ }^{\mathrm{c}}$, B. Nellen ${ }^{\mathrm{c}}$,

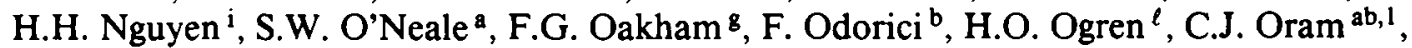
M.J. Oreglia ${ }^{i}$, S. Orito ${ }^{x}$, J.P. Pansart ${ }^{\text {u }}$, B. Panzer-Steindel ${ }^{\text {h }}$, P. Paschievici ${ }^{z}$, G.N. Patrick ${ }^{\text {, }}$ N. Paz-Jaoshvili ${ }^{w}$, M.J. Pearce ${ }^{a}$, P. Pfister ${ }^{j}$, J.E. Pilcher ${ }^{i}$, J. Pinfold ${ }^{\text {ad }}$, D. Pitman ${ }^{\text {ab }}$, D.E. Plane ${ }^{\mathrm{h}}$, P. Poffenberger ${ }^{\mathrm{ab}}$, B. Poli ${ }^{\mathrm{b}}$, T.W. Pritchard ${ }^{\mathrm{m}}$, H. Przysiezniak ${ }^{\mathrm{r}}$, G. Quast ${ }^{\mathrm{aa}}$, M.W. Redmond ${ }^{\text {h }}$, D.L. Rees ${ }^{\text {h }}$, G.E. Richards ${ }^{p}$, M. Rison ${ }^{e}$, S.A. Robins ${ }^{e}$, D. Robinson ${ }^{\text {h }}$, A. Rollnik ${ }^{\text {c }}$, J.M. Roney ${ }^{\text {ab }}$, E. Ros ${ }^{\text {b }}$, S. Rossberg ${ }^{\text {j }}$, A.M. Rossi ${ }^{b}$, M. Rosvick ${ }^{\text {ab }}$, P. Routenburg ${ }^{\text {ad }}$, K. Runge ${ }^{j}$, O. Runolfsson ${ }^{b}$, D.R. Rust ${ }^{\ell}$, M. Sasaki ${ }^{x}$, C. Sbarra ${ }^{b}$, A.D. Schaile ${ }^{z}$, O. Schaile ${ }^{j}$, W. Schappert ${ }^{f}$, F. Scharf ${ }^{c}$, P. Scharff-Hansen ${ }^{\text {h }}$, P. Schenk ${ }^{d}$, B. Schmitt ${ }^{\text {c }}$, H. von der Schmitt ${ }^{k}$, 
M. Schröder ${ }^{\ell}$, C. Schwick ${ }^{\text {aa }}$, J. Schwiening ${ }^{c}$, W.G. Scott ${ }^{t}$, M. Settles ${ }^{\ell}$, T.G. Shears ${ }^{\mathrm{e}}$, B.C. Shen ${ }^{d}$, C.H. Shepherd-Themistocleous ${ }^{8}$, P. Sherwood ${ }^{\circ}$, G.P. Siroli ${ }^{b}$, A. Skillman ${ }^{p}$, A. Skuja ${ }^{q}$, A.M. Smith ${ }^{\text {h }}$, T.J. Smith ${ }^{\text {ab }}$, G.A. Snow ${ }^{q}$, R. Sobie ${ }^{a b}$, R.W. Springer ${ }^{q}$, M. Sproston ${ }^{t}$, A. Stahl ${ }^{\text {c }}$, C. Stegmann ${ }^{\text {j, K. Stephens }}{ }^{\text {p }}$, J. Steuerer ${ }^{\text {ab }}$, R. Ströhmer ${ }^{\text {k }}$, D. Strom ${ }^{\text {s }}$, H. Takeda ${ }^{x}$, T. Takeshita ${ }^{x, 3}$, S. Tarem $^{z}$, M. Tecchio ${ }^{i}$, P. Teixeira-Dias ${ }^{k}$, N. Tesch ${ }^{c}$, M.A. Thomson ${ }^{\circ}$, E. Torrente-Lujan ${ }^{v}$, S. Towers ${ }^{a b}, G$. Transtromer ${ }^{y}$, N.J. Tresilian ${ }^{p}$, T. Tsukamoto ${ }^{x}$, M.F. Turner ${ }^{\mathrm{h}}$, D. Van den plas ${ }^{\mathrm{r}}$, R. Van Kooten ${ }^{\text {aa }}$, G.J. VanDalen ${ }^{\mathrm{d}}$, G. Vasseur ${ }^{\mathrm{u}}$, A. Wagner ${ }^{\text {aa }}$, D.L. Wagner ${ }^{i}$, C. Wahl ${ }^{j}$, C.P. Ward ${ }^{e}$, D.R. Ward ${ }^{\text {e }}$, P.M. Watkins ${ }^{\text {a }}$, A.T. Watson ${ }^{\text {a }}$, N.K. Watson ${ }^{h}$, M. Weber ${ }^{k}$, P. Weber ${ }^{f}$, P.S. Wells ${ }^{h}$, N. Wermes ${ }^{c}$, M.A. Whalley ${ }^{\mathrm{a}}$, B. Wilkens ${ }^{j}$, G.W. Wilson ${ }^{d}$, J.A. Wilson ${ }^{a}$, V-H. Winterer ${ }^{j}$, T. Wlodek ${ }^{2}$, G. Wolf ${ }^{z}$, S. Wotton ${ }^{k}, T . R$. Wyatt $^{p}$, R. Yaari ${ }^{z}$, A. Yeaman ${ }^{\mathrm{m}}$, G. Yekutieli ${ }^{\mathrm{z}}$, M. Yurko ${ }^{\mathrm{r}}$, W. Zeuner ${ }^{\mathrm{h}}$ and G.T. Zorn $^{\mathrm{q}}$.

a School of Physics and Space Research, University of Birmingham, Birmingham, B15 2TT, UK

b Dipartimento di Fisica dell' Università di Bologna and INFN, Bologna, 40126, Italy

c Physikalisches Institut, Universität Bonn, D-5300 Bonn 1, Germany

d Department of Physics, University of California, Riverside, CA 92521, USA

e Cavendish Laboratory, Cambridge, CB3 OHE, UK

f Carleton University, Department of Physics, Colonel By Drive, Ottawa, Ontario KIS 5B6, Canada

- Centre for Research in Particle Physics, Carleton University, Ottawa, Ontario KIS 5B6, Canada

h CERN. European Organisation for Particle Physics, 1211 Geneva 23, Switzerland

i Enrico Fermi Institute and Department of Physics, University of Chicago, Chicago, IL 60637, USA

j Fakultät für Physik, Albert Ludwigs Universität, D-7800 Freiburg, Germany

k Physikalisches Institut, Universität Heidelberg, Heidelberg, Germany

$\ell$ Indiana University, Department of Physics, Swain Hall West 117, Bloomington, IN 47405, USA

m Queen Mary and Westfield College, University of London, London, EI 4NS, UK

${ }^{n}$ Birkbeck College, London, WCIE $7 H V$. UK

- University College London, London, WCIE 6BT, UK

p Department of Physics, Schuster Laboratory, The University, Manchester, MI3 9PL, UK

q Department of Physics, University of Maryland. College Park, MD 20742, USA

' Laboratoire de Physique Nucléaire, Université de Montréal, Montréal, Quebec H3C 3J7, Canada

- University of Oregon, Department of Physics, Eugene, OR 97403. USA

' Rutherford Appleton Laboratory, Chilton, Didcot, Oxfordshire, OXI1 0QX, UK

"DAPNIA/SPP, Saclay, F-91191 Gif-sur-Yvette, France

v Department of Physics. Technion-Israel Institute of Technology, Haifa 32000, Israel

" Department of Physics and Astronomy, Tel Aviv University, Tel Aviv 69978, Israel

* International Centre for Elementary Particle Physics and Department of Physics, University of Tokyo. Tokyo 113, Japan and Kobe University, Kobe 657, Japan

y Brunel University, Uxbridge, Middlesex, UB8 $3 P H, U K$

${ }^{2}$ Nuclear Physics Department, Weizmann Institute of Science, Rehovot 76100, Israel

an Universität Hamburg/DESY, II Institut für Experimental Physik, D-2000 Hamburg 52, Germany

ab University of Victoria, Department of Physics, P O Box 3055, Victoria, BC V8W 3P6, Canada

ac University of British Columbia, Department of Physics, Vancouver, BC V6T IZI, Canada

ad University of Alberta, Department of Physics, Edmonton, AB T6G 2N5, Canada

ac Duke University, Department of Physics, Durham, NC 27708-0305, USA

Received 11 August 1993

Editor: K. Winter

The average lifetime of baryons containing a $b$ quark has been measured with the OPAL detector using data collected between 1990 and 1992. Semileptonically decaying b-flavoured baryons which produce a $\Lambda$ are identified through the charge correlation of the $\Lambda$ and lepton. The decay point of the $b$ baryon is estimated by the $\Lambda$-lepton vertex, and the observed distribution of decay lengths is fitted to determine the average lifetime. In the data sample consisting of 261 right-sign and 104 wrong-sign $\Lambda$-lepton charge combinations, the average $b$ baryon lifetime is found to be $\tau=1.05_{-0.20}^{+0.23} \pm 0.08 \mathrm{ps}$, where the first error is statistical and the second systematic. 


\section{Introduction}

The $b$ quark lifetime is important for determining the CKM mixing parameter $V_{\mathrm{cb}}$. The estimation of heavy quark lifetimes from the observed weakly decaying hadron lifetimes is complicated by the influence of the other quarks in the hadron, called spectator quarks, in the decay process. For example, in the charm system the lifetime of the baryon $\Lambda_{c}^{+}$is about five times shorter than that of the charged meson $\mathrm{D}^{+}$. Because the $b$ quark is much heavier than the $c$ quark, the influence of the spectator quarks is thought to be much smaller in $b$ hadron decays. As a result, lifetimes of the different weakly decaying b-flavoured hadrons are expected to be equal to within $10-20 \%$ [1]. It is important to verify this by direct measurements of individual $b$ hadron lifetimes.

Experiments at the LEP collider have provided the most precise measurements of the average $b$ hadron lifetime [2] and several exclusive $b$ hadron lifetimes [3-9]. This paper describes the measurement of the average $b$ baryon lifetime using the OPAL detector, by studying events which contain decay chains of the form

$$
\begin{array}{rlrl}
\Lambda_{\mathrm{b}}^{0} \rightarrow \Lambda_{\mathrm{c}}^{+} \ell^{-} \bar{\nu} X & & \Xi_{\mathrm{b}}^{0} \rightarrow & \Xi_{\mathrm{c}}^{+} \ell^{-} \bar{\nu} X \\
\hookrightarrow \Lambda X & & \\
\hookrightarrow \mathrm{p} \pi^{-} & & & \\
& & & \hookrightarrow X \\
& & \hookrightarrow \mathrm{p} \pi^{-}
\end{array} .
$$

Charge conjugate processes are implied throughout the paper. It is expected that more $\Lambda_{b}^{0}$ (quark content: bud) are produced than $\Xi_{\mathrm{b}}^{0}$ (bsu) and $\Xi_{\mathrm{b}}^{-}$(bsd), and hence the symbol $\Lambda_{b}$ will be used in this paper to refer to all $b$ baryons. This analysis measures the average lifetime of weakly decaying $b$ baryons weighted by the different production rates, branching ratios, and detection efficiencies of the various $b$ baryons. The lifetime of the neutral $\mathbf{b}$ baryons are expected to be about $10 \%$ shorter than the charged $b$ baryons [10].

The decay length between the primary event vertex and the vertex formed by the lepton and $\Lambda$ is used to estimate the flight distance of the $b$ baryon. The fact that the lepton and the $\Lambda$ do not originate from the same vertex turns out not to be important in this analysis for two reasons. Firstly, the lifetime

\footnotetext{
Also at TRIUMF, Vancouver, Canada V6T $2 \mathrm{~A} 3$.

Now at MPI, München, Germany.

Also at Shinshu University, Matsumoto 390, Japan.
}

of the charmed baryons [11] $\left(\tau_{\Lambda_{\mathrm{c}}^{+}}=0.19 \pm 0.02 \mathrm{ps}\right.$, $\left.\tau_{\Xi_{c}^{+}}=0.3 \pm 0.1 \mathrm{ps}\right)$ are much smaller than the average $b$ hadron lifetime. Also, since the difference between the charmed and the strange baryon masses is much smaller than the mass difference between the $\mathrm{b}$ and $\mathrm{c}$ quark, the transverse momentum of the $\Lambda$ with respect to the charmed baryon direction is typically much smaller than the transverse momentum of $b$ hadron decay products. As a result of these two effects, the impact parameter of the $\Lambda$ with respect to the $b$ baryon decay point is small.

In the following sections, the OPAL detector and the event selection are briefly described. Then the decay length estimator and the fitting procedure are defined. After checks of the method with Monte Carlo data, the result of the fit for the lifetime of $b$ baryons in the data is given and estimates of systematic uncertainties are discussed.

\section{The OPAL detector}

Only a brief description of the OPAL detector is given here; complete details are found elsewhere [12]. Tracking of charged particles is performed using the three gaseous drift chambers which form the central detector - a precision vertex drift chamber, a large volume jet chamber, and a set of chambers that measures the $z$-coordinate ${ }^{\# 1}$ of tracks as they leave the jet chamber. The central detector is located inside a solenoid which provides a magnetic field of $0.435 \mathrm{~T}$. Before the data taking period began in 1991, a silicon microvertex chamber was installed and became operational throughout most of that year and for all of 1992. Information from this detector, however, is not used in this analysis so that the tracking resolution would be uniform throughout the entire data sample.

The jet chamber also helps to identify charged particles by measuring their ionization loss as they travel through the chamber [13]. The lead-glass electromagnetic calorimeter with presampler, surrounding the central detector and solenoid, is used in this analysis for hadronic event selection, jet reconstruction, and electron identification. Outside the instrumented iron

\#1 The OPAL coordinate system is defined with positive $z$ being along the electron beam direction, $\theta$ and $\phi$ being the polar and azimuthal angles, respectively. 
return yoke of the magnet which forms the hadron calorimeter are chambers used for muon identification.

\section{Event selection}

The analysis presented in this paper uses the data sample collected between 1990 and 1992. Hadronic $Z^{0}$ decays are selected as described elsewhere [14], and events from this sample which have at least seven "good" charged tracks are used in the analysis. Good tracks are those which are reconstructed using at least 20 jet chamber hits with a $\chi^{2}$ per degree of freedom from the track fit in the $x-y$ plane less than 100 , have a momentum less than $65 \mathrm{GeV} / c$, and have a momentum in the $x-y$ plane greater than $0.15 \mathrm{GeV} / c$. In addition, the distance of closest approach to the nominal beam axis must be less than $5 \mathrm{~cm}$, and the $z$ coordinate of this point must be within $50 \mathrm{~cm}$ of the interaction point. Only the data in which the tracking chambers, electromagnetic calorimeters, barrel presampler, and muon detectors were operating properly are used in this analysis. After these track quality and detector performance requirements, 1234203 hadronic $Z^{0}$ decay events are selected.

The identification of $b$ baryon decays using charge correlated $\Lambda$-lepton combinations closely follows the analysis previously reported by OPAL [15]. Electron candidates are identified using their ionisation loss measured in the jet chamber and their shower shapes from the electromagnetic calorimeter and presampler $[16,17]$. Muons are found by associating track segments in the outer muon detectors with tracks in the central detector $[18,17]$. Electron and muon candidates are required to pass the good track criteria defined above.

All charged tracks and those electromagnetic clusters with no associated track are clustered into jets using the JADE algorithm [19] with the E0 recombination scheme [20] that has the invariant mass-squared cut-off set to $x_{\min }=49 \mathrm{GeV}^{2}$. In order to obtain an analysis sample enriched in b quark events, lepton candidates are required to have momentum $p>$ $3 \mathrm{GeV} / c$ and transverse momentum with respect to the associated jet $p_{t}>0.8 \mathrm{GeV} / c$. The lepton is included in the calculation of the jet direction.

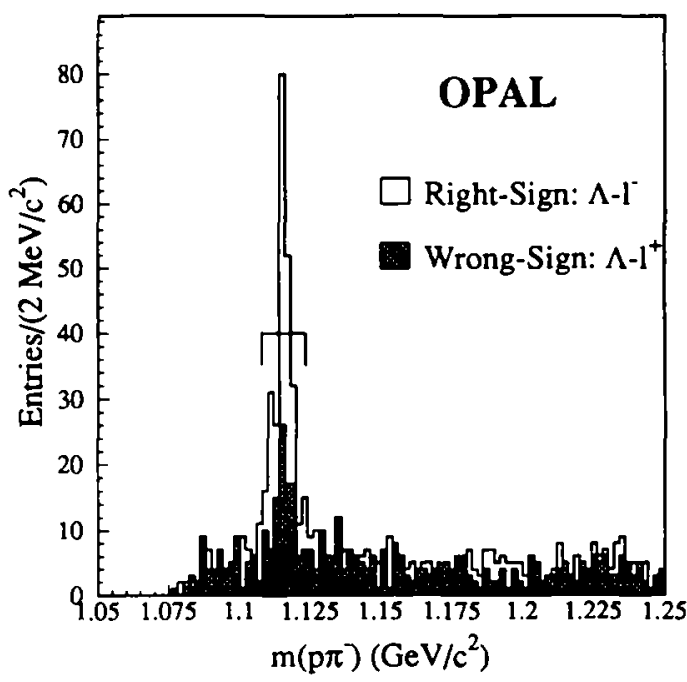

Fig. 1. $\mathrm{p} \pi$ invariant mass distribution for $\Lambda \ell^{-}$(open histogram) superimposed by $\Lambda \ell^{+}$(shaded histogram) correlations for the total data sample. The signal region is shown by the bracketed range of masses. The peak at the $\Lambda$ mass in the wrong-sign mass is indicative of genuine $\Lambda$ particles (though not necessarily from $b$ baryon decay) being combined with fake leptons (i.e. hadrons misidentified as leptons).

$\Lambda$ candidates are identified via the decay $\Lambda \rightarrow p \pi^{-}$. To reduce combinatorial backgrounds, the distance between the $\Lambda$ vertex position and the beam axis is required to be greater than $5 \mathrm{~cm}$. It is further demanded that $\left|\phi_{\Lambda}\right|<14 \mathrm{mrad}$, where $\phi_{\Lambda}$ is the angle in the $x-y$ plane between the reconstructed $\Lambda$ momentum vector and the line joining the primary and $\Lambda$ decay vertices. The track with the larger momentum is assumed to be the proton, and its ionisation loss measured in the jet chamber is required to be consistent with that of a proton. The other track, assumed to be a pion, must have a distance of closest approach to the beam axis of greater than $1 \mathrm{~mm}$, to reduce combinatorial backgrounds. To reduce the contribution of $\Lambda$ 's from fragmentation processes, $\Lambda$ candidates are required to have $p_{\Lambda}>4 \mathrm{GeV} / c$.

To select events containing a b baryon, a $\Lambda$ candidate is required to be within a cone of half-angle $50^{\circ}$ about a lepton candidate. Additional cuts of $m_{\Lambda \ell}>$ $2.2 \mathrm{GeV} / c^{2}$, where $m_{\Lambda \ell}$ is the invariant mass of the $\Lambda \ell$ pair, and $p_{\Lambda \ell}>9 \mathrm{GeV} / c$, where $p_{\Lambda \ell}$ is the magnitude of the vector sum of the $\Lambda$ and lepton momenta, are made to reduce backgrounds. After these cuts, the 
$\mathrm{p} \pi$ invariant mass distributions for the $\Lambda \ell^{-}$(rightsign) and for the $\Lambda \ell^{+}$(wrong-sign) combinations are as shown in fig. 1. A signal region is defined for $\Lambda$ candidates with invariant mass in the range $1.1078<$ $m_{\mathrm{p} \pi}<1.1234 \mathrm{GeV} / c^{2}$. A total of 261 right-sign and 104 wrong-sign $\Lambda$-lepton charge combinations are selected. The 157 excess right-sign charge combinations are attributed to $b$ baryon decays.

\section{Monte Carlo event samples}

Approximately 1.1 million events simulating hadronic decays of the $Z^{0}$ were generated by the JETSET 7.3 program [21] and are used to check the analysis. Approximately half of the sample has heavy quark fragmentation according to Peterson fragmentation [22] and the remaining are with LUND symmetric fragmentation [21]. Three additional samples enriched in b baryons were created to estimate systematic errors and are referred to as the "standard", "EURODEC", and "polarization" samples. The "standard" enriched Monte Carlo sample, generated with JETSET 7.3, corresponds to roughly $1 \mathrm{mil}-$ lion $Z^{0} \rightarrow b \bar{b}$ events. In the "EURODEC" sample, roughly 5 times larger, the only $b$ baryon included is the $\Lambda_{b}^{0}$. It was generated with JETSET 7.3, used EURODEC [23] routines for $\Lambda_{c}$ decays with branching ratios adjusted to agree with the current measured branching fractions [11], and included b baryon decay form factors [24] calculated to first order in the inverse of the $b$ quark mass. The "polarization" sample was generated assuming the $b$ quark polarization is completely transferred to the b baryon [25] to estimate the sensitivity for the lifetime measurement. All of these events were processed by a detector simulation program [26] which includes a detailed description of the detector geometry and material as well as effects of detector resolutions and efficiencies.

\section{Definition of the decay length}

The flight length of the $b$ baryon is estimated using the positions and error matrices of the primary vertex and the $\Lambda$-lepton vertex subject to a directional constraint. The primary vertex is found by using the tracks in the event (excluding the candidate lepton, proton

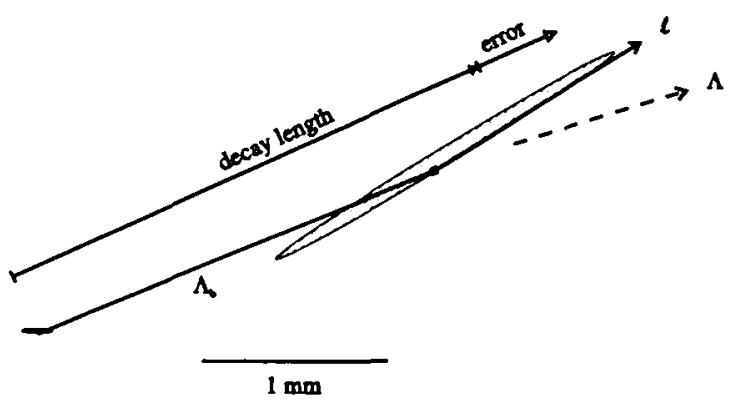

Fig. 2. The method of determining the decay length is illustrated for a typical Monte Carlo event, viewed in the plane perpendicular to the beam. The small and large ellipses represent the one standard deviation error ellipses of the primary vertex and $\Lambda$-lepton vertex, respectively. The decay length determined using the lepton-jet direction is shown along with its error on a line along this direction. The true paths of the $b$ baryon, the lepton and the $\Lambda$ are overlaid. The major axis of the secondary vertex is aligned with the lepton direction since it is more precisely measured than the $\Lambda$ direction.

and pion tracks) as well as the LEP fill-averaged beam position [27]. The decay length in the plane perpendicular to the beam is defined by the length of the line parallel to the jet containing the lepton whose endpoints minimise the $\chi^{2}$ formed from the deviations of these endpoints from the primary and secondary vertices. This is then converted into a three dimensional decay length by dividing by the sine of the polar angle of the momentum sum of the $\Lambda$ and lepton. A typical Monte Carlo event is shown in fig. 2 to demonstrate the method. Monte Carlo studies of the resolution of the decay length indicate that use of the directional constraint, rather than just the simple primary to secondary vertex distance, reduces the RMS width of the residuals from $4.4 \mathrm{~mm}$ to $2.9 \mathrm{~mm}$. This is due partly to a reduction in the Gaussian width of the central core from $1.1 \mathrm{~mm}$ to $0.96 \mathrm{~mm}$, but mainly from a large reduction in the tails of the distribution.

\section{Fit of the observed decay length distribution}

A simultaneous fit to the decay length distributions of the right-sign and wrong-sign $\Lambda$-lepton combinations is performed to determine the average $b$ baryon lifetime. Since the decay length distribution of the background processes are expected to be independent 
of the sign correlation of the $\Lambda$-lepton combinations, the wrong-sign sample is used to define the background shape in the right-sign sample.

\subsection{Model for the decay length distribution of signal events}

For a particle with lifetime $\tau$, the probability density function (PDF) for an observed proper decay time, $t$, with associated uncertainty, $\sigma_{t}$, is given by the convolution of an exponential distribution with a Gaussian distribution:

$$
\begin{aligned}
\mathcal{C} & \left(\tau, t, \sigma_{t}\right) \\
& =\int_{0}^{\infty} \frac{\exp \left[-\left(t-t^{\prime}\right)^{2} / 2 \sigma_{t}^{2}\right]}{\sqrt{2 \pi} \sigma_{t}} \frac{\exp \left(-t^{\prime} / \tau\right)}{\tau} \mathrm{d} t^{\prime} \\
& =\frac{1}{2 \tau} \exp \left(\frac{\sigma_{t}^{2}}{2 \tau^{2}}-\frac{t}{\tau}\right) \operatorname{erfc}\left(\frac{\sigma_{t}}{\sqrt{2} \tau}-\frac{t}{\sqrt{2} \sigma_{t}}\right) .
\end{aligned}
$$

However, our measurements are of the decay length, $l$, and its uncertainty, $\sigma_{l}$. Therefore, we use the relation between the decay length and proper decay time,

$l=\frac{p_{\Lambda_{b}}}{m_{\Lambda_{b}}} t$,

and convolute the PDF (2) with a distribution of $b$ baryon momenta, $p_{\Lambda_{b}}$, as described in the next section.

\subsubsection{Estimation of the b baryon momentum spectrum}

The $b$ baryon momentum distribution is formed from the observed $\Lambda$ and lepton momenta in the data in order to be less sensitive to $b$ quark fragmentation uncertainties. For any event the true b baryon momentum falls between the values of

$p_{\Lambda \ell}=\left|p_{\Lambda}+p_{\ell}\right|$ and $p_{\Lambda_{\mathrm{b}} \max }=\sqrt{E_{\text {beam }}^{2}-m_{\Lambda_{\mathrm{b}}}^{2}}$.

The "standard" b baryon enriched Monte Carlo sample is used to determine the $b$ baryon momentum distribution within the two limits mapped to $y \epsilon(0,1)$. In other words, the function $g(y)$ is found which is the PDF of the quantity $y$, where

$y=\frac{p_{\Lambda_{b}}-p_{\Lambda \ell}}{p_{\Lambda_{b} \max }-p_{\Lambda \ell}}$.
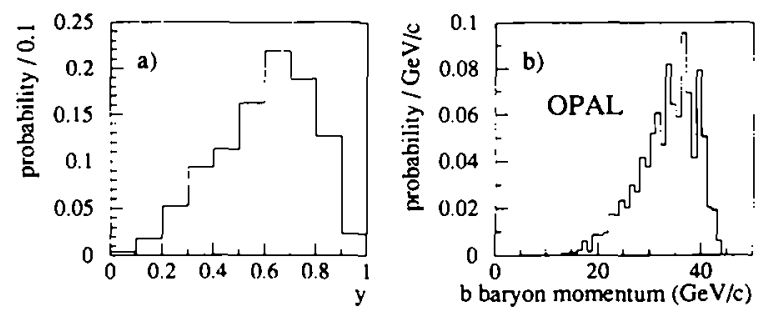

Fig. 3. (a) The distribution of the variable, $y$, as determined from the "standard" b baryon Monte Carlo sample. (b) The estimate of the b baryon momentum spectrum of the data using the procedure described in the text.

In the Monte Carlo sample, the distribution for $g(y)$ is seen not to depend strongly on $p_{\Lambda \ell}$. The $\mathrm{b}$ baryon momentum distribution in the data is estimated from the convolution of the observed $p_{\Lambda}$ spectrum with the Monte Carlo determined $g(y)$ distribution. Taking $p_{\Lambda \ell}$ from Monte Carlo reconstructed tracks ensures that momentum resolution effects are taken into account in the formation of the estimated $b$ baryon momentum spectrum. A further step is taken to remove the effect of the background by subtracting the estimated $b$ baryon momentum spectrum of the wrongsign combinations from that of the right-sign combinations. This procedure is found to reproduce the original Monte Carlo momentum distribution well and results in similar b baryon spectra for the data and Monte Carlo samples. Fig. 3 shows the $g(y)$ function determined from the $b$ baryon Monte Carlo sample and the resulting estimate of the $b$ baryon momentum spectrum for the data.

\subsubsection{Definition of the signal PDF}

Once the b baryon momentum spectrum is determined, as described above, it is normalized and binned into $1 \mathrm{GeV} / \mathrm{c}$ bins, as shown in fig. $3 \mathrm{~b}$, with the central value and content of bin $i$ given by $p_{\Lambda_{\mathrm{b}}}^{i}=$ (i $-\frac{1}{2}$ ) GeV/c and $h\left(p_{\Lambda_{\mathrm{b}}}^{i}\right)$, respectively. Then, the PDF for decay lengths of the $b$ baryon signal events is found by the convolution

$\mathcal{S}\left(\tau, l, \sigma_{l}\right)=\sum_{i=1}^{50} h\left(p_{\Lambda_{\mathrm{b}}}^{i}\right) \mathcal{C}\left(\tau, \frac{m_{\Lambda_{\mathrm{b}}}}{p_{\Lambda_{\mathrm{b}}}^{i}} l, \frac{m_{\Lambda_{\mathrm{b}}}}{p_{\Lambda_{\mathrm{b}}}^{i}} \sigma_{l}\right)$,

where $\mathcal{C}$ is defined in eq. (2). In order to reduce the sensitivity of the fit to the decay length errors and 
to allow for occasional mismeasurements, the signal PDF actually used in this analysis is

$$
\begin{aligned}
& \mathcal{P}_{\text {sig }}\left(\tau, l, \sigma_{l}, k_{1}, f_{2}, k_{2}\right) \\
& =\left(1-f_{2}\right) \mathcal{S}\left(\tau, l, k_{1} \sigma_{l}\right)+f_{2} \mathcal{S}\left(\tau, l, k_{2} \sigma_{l}\right),
\end{aligned}
$$

where $k_{1}$ is an error scale factor for the majority of measurements and $f_{2}$ is the fraction of events which are mismeasured with a corresponding larger scale factor $k_{2}$. This form thus describes the decay length resolution function in terms of the sum of two Gaussian distributions, which allows for resolution tails. Since the parameters $k_{1}, f_{2}$, and $k_{2}$ are unknown, they are allowed to vary in the lifetime fit. If the parameters $f_{2}$ and $k_{2}$ were not included, it would be necessary to restrict the range of decay lengths which are used in the fit, in order to reduce the potential bias from badly measured decay lengths. By allowing $f_{2}$ and $k_{2}$ to vary in the fit, all decay lengths are used and the statistical precision of the lifetime estimate is unaffected.

\subsection{Model for the decay length distribution of background events}

The decay length distribution for background events is parametrised in terms of an exponential and a $\delta$-function at zero (to account for background events with and without lifetime, respectively), convolved by a Gaussian distribution with errors given by the measured decay length error,

$$
\begin{aligned}
\mathcal{B} & \left(l, \sigma_{l}, L_{+}, b_{+}, b_{0}\right) \\
& =\frac{1}{b_{+}+b_{0}}\left[b_{+} \mathcal{C}\left(L_{+}, l, \sigma_{l}\right)+b_{0} \mathcal{G}\left(l, \sigma_{l}\right)\right],
\end{aligned}
$$

where $L_{+}$is the characteristic length of the lifetime exponential, and $b_{+}\left(b_{0}\right)$ is the fraction of events in the right-sign sample which come from background events with (without) lifetime. The function $\mathcal{C}$ is defined in eq. (2) and $\mathcal{G}\left(l, \sigma_{l}\right)$ is the Gaussian PDF of mean 0 and standard deviation $\sigma_{l}$. As in the signal decay length distribution, the possibility is included for a scale factor on the estimated error and also for a fraction of events to have a larger scale factor. The background PDF is thus given by

$$
\begin{aligned}
& \mathcal{P}_{\text {back }}\left(l, \sigma_{l}, L_{+}, b_{+}, b_{0}, \tilde{k}_{1}, \tilde{f}_{2}, \tilde{k}_{2}\right) \\
& =\left(1-\tilde{f}_{2}\right) \mathcal{B}\left(l, \tilde{k}_{1} \sigma_{l}, L_{+}, b_{+}, b_{0}\right) \\
& +\tilde{f}_{2} \mathcal{B}\left(l, \tilde{k}_{2} \sigma_{l}, L_{+}, b_{+}, b_{0}\right) .
\end{aligned}
$$

The terms $\widetilde{k}_{1}, \widetilde{f}_{2}$, and $\widetilde{k}_{2}$ are included in order to make the background parametrisation as general as possible. Given the variety of background sources, these parameters should not be understood as a measure of the detector resolution.

The number of background events in the rightsign sample is constrained by the observed number of events in the wrong-sign sample. Given $N$ total $\Lambda$-lepton candidates, and the probability, $p_{w s}$, that a $\Lambda$-lepton candidate has the wrong-sign correlation, the PDF for the number of events in the wrong-sign sample $N_{\text {ws }}$ is given by the binomial PDF,

$$
\begin{aligned}
& \mathcal{P}\left(N_{\mathrm{ws}}, N, p_{\mathrm{ws}}\right)=\frac{N !}{N_{\mathrm{ws}} !\left(N-N_{\mathrm{ws}}\right) !} \\
& \times p_{\mathrm{ws}}^{N_{\mathrm{ws}}}\left(1-p_{\mathrm{ws}}\right)^{N-N_{\mathrm{ws}}} .
\end{aligned}
$$

Given the definition of $b_{+}$and $b_{0}$, the wrong-sign probability is $p_{w s}=\left(b_{+}+b_{0}\right) /\left(1+b_{+}+b_{0}\right)$.

\subsection{Combined fit of the decay length distributions}

The decay length PDF for the right-sign $\Lambda$-lepton combinations is described by the sum of the signal and background PDF's, $\mathcal{P}_{\text {sig }}$ and $\mathcal{P}_{\text {back }}$, whereas the wrongsign combinations follow $\mathcal{P}_{\text {back }}$ alone. The unbinned likelihood function is thus defined to be the product of the values of the probability density functions for each observed $\Lambda$-lepton combination multiplied by the binomial distribution, given above. Hence the log likelihood is given by

$$
\begin{aligned}
& \log \mathcal{L}=\sum_{i}^{\text {(right) }} \log \left[\left(1-b_{+}-b_{0}\right) \mathcal{P}_{\text {sig }}+\left(b_{+}+b_{0}\right) \mathcal{P}_{\text {back }}\right] \\
& +\sum_{j}^{\text {(wrong) }} \log \mathcal{P}_{\text {back }}+N_{\text {wrong }} \log \left(\frac{b_{+}+b_{0}}{1+b_{+}+b_{0}}\right) \\
& +N_{\text {right }} \log \left(\frac{1}{1+b_{+}+b_{0}}\right)
\end{aligned}
$$

where the sum over $i$ runs over all the right-sign $\Lambda$ lepton combinations and the sum over $j$ runs over all the wrong-sign combinations. The negative log likelihood function is minimised by allowing the lifetime 
$\tau$, the error scale factor parameters $\left(k_{1}, f_{2}, k_{2}\right)$, and the background parameters $\left(L_{+}, b_{+}, b_{0}, \widetilde{k}_{1}, \widetilde{f}_{2}, \widetilde{k}_{2}\right)$ to vary.

\subsection{Checks of the fitting procedure with Monte Carlo data}

The mean lifetime of the $800 \Lambda$-lepton combinations from b baryon decays in the "standard" enriched Monte Carlo sample which pass the event selection and are properly identified is $1.22 \pm 0.04$ ps. The result of the fit to the decay length distribution from this sample is $1.18 \pm 0.06 \mathrm{ps}$. This result verifies the statement made in the introduction, that the non-zero lifetime of the charmed baryon does not significantly bias the lifetime estimator. The result is stable within $0.04 \mathrm{ps}$ if the following changes to the analysis are made: Only candidates with decay lengths between -1 and $2 \mathrm{~cm}$ are used; only candidates with decay length errors less than $5 \mathrm{~mm}$ are used; only candidates with the $\chi^{2}$ of the length calculation less than 10 are used; or the track parameters are smeared by an additional $30 \%$.

A further check is made that no bias exists in the fitting procedure and that the statistical error estimates are valid with a simple Monte Carlo program. The program was used to produce 200 data samples with decay lengths given by an exponential lifetime distribution smeared by errors following the error distribution seen in the data, and a background distribution according to the model described above. The samples have an average of 360 events each and were generated with the model parameters similar to the results from the fit to the data. The mean lifetime determined from the fits of the samples is $0.019 \pm 0.014$ ps below the input lifetime and the average lifetime uncertainty is $0.18 \mathrm{ps}$. The distribution of lifetime residuals divided by errors is described by a Gaussian distribution with $\sigma=1.05 \pm 0.10$, indicating the statistical error to be correctly evaluated.

These samples are also used to define a goodnessof-fit test. The range of decay lengths between $-1 \mathrm{~cm}$ and $2 \mathrm{~cm}$ is divided into 11 unequal size bins for the right-sign and 5 bins for the wrong-sign candidates, chosen so that the expectation in all bins is greater than 5 events. The variable, $X^{2}=\sum_{i}\left(n_{i}-m_{i}\right)^{2} / m_{i}$, is formed. Here, $n_{i}$ is the number of observed events in bin $i$ and $m_{i}$ is the number expected from the model once the unknown parameters are determined in the fit. The $X^{2}$ distributions approximately follow $\chi^{2}$ distributions with 8 and 3 degrees of freedom for the right and wrong-sign distributions, respectively. This empirical method is the only way to estimate the probability distribution for the $X^{2}$ variable. The total number of degrees of freedom does not correspond to the total number of bins minus the number of parameters because the model is not linear in these parameters and because the parameters are not estimated with a least squares method. In the results below, the value of $X^{2}$ from the fit is referred to as the " $\chi^{2}$ of the fit".

The complete analysis was applied to the Monte Carlo sample of 1.1 million hadronic events, a size roughly equivalent to the data sample. A total of 291 right-sign and 123 wrong-sign $\Lambda$-lepton combinations are selected and the estimated lifetime from the fit, $\tau=1.31_{-0.21}^{+0.23} \mathrm{ps}$, is in good agreement with the input lifetime of $1.3 \mathrm{ps}$.

\section{Result of lifetime fit of the data}

The lifetime is measured using all events in the data which pass the $\Lambda$-lepton selection with no additional quality cuts applied. The result of the fit to the decay length distribution is shown in fig. 4 , which finds $\tau=0.93_{-0.18}^{+0.20} \mathrm{ps}$. The $\chi^{2}$ of the fit is 7.7 and 1.9 for the right-sign and wrong-sign candidates for 8 and 3 degrees of freedom, respectively. The values of the other parameters in the fit are shown in table 1.

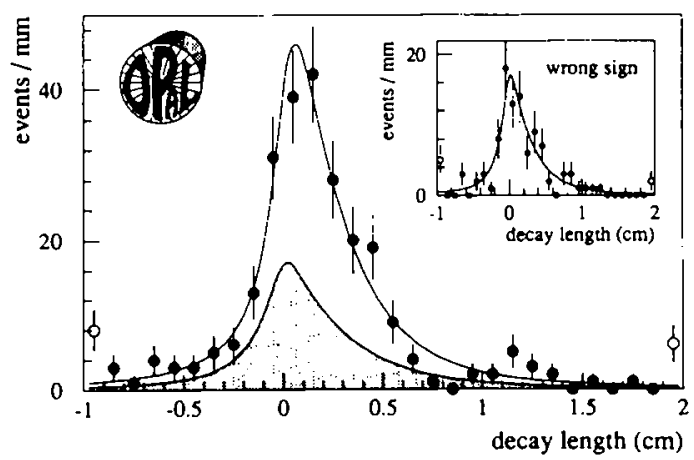

Fig. 4. The distribution of decay lengths observed in the data are shown along with the result of the fit. The inset figure shows the distribution of the wrong-sign candidates. The underflow and overflow entries, shown in the first and last bins, have large decay length errors. 
Table 1

The model parameters found in the likelihood fit to the full data set.

\begin{tabular}{lllllllll}
\hline$k_{1}$ & $f_{2}$ & $k_{2}$ & $b_{+}$ & $b_{0}$ & $L_{+}(\mathrm{cm})$ & $\tilde{k}_{1}$ & $\tilde{f}_{2}$ & $\tilde{k}_{2}$ \\
\hline $1.2 \pm 0.3$ & $0.26 \pm 0.18$ & $3.9 \pm 1.2$ & $0.22 \pm 0.06$ & $0.17 \pm 0.05$ & $0.24 \pm 0.06$ & $2.1 \pm 0.3$ & $0.10 \pm 0.05$ & $11.0 \pm 3.0$ \\
\hline
\end{tabular}

The log likelihood function is found to be approximately parabolic. By fixing the lifetime at various points, and allowing the other parameters to vary to maximise the likelihood, the 1,2 , and 3 standard deviation points are found to occur at $(-0.18,+0.20)$, $(-0.33,+0.42)$ and $(-0.47,+0.67)$ ps away from the central value of $0.93 \mathrm{ps}$.

\section{Estimates of systematic uncertainties}

Some systematic uncertainties are already accounted for in the statistical error because of the free parameters which are allowed to vary in the fit. This is the case for the uncertainty in the intrinsic decay length resolution as well as the uncertainties in the shape and normalisation of the background. This procedure increases the statistical error by about $0.04 \mathrm{ps}$ as compared to a fit which allows only the lifetime to vary (which corresponds to an additional error of about 0.1 ps added in quadrature).

Other possible systematic effects are considered in the following sections and are summarised in table 2. The total systematic correction increases the fit lifetime by $13 \%$, and hence the statistical errors from the fit are increased by the same amount. Since the different sources of systematic uncertainties are expected to be independent, the total systematic error is found by adding the individual errors in quadrature, giving $0.08 \mathrm{ps}$.

\subsection{Bias of the decay length estimator}

An estimate of a possible bias of the lifetime from the decay length estimator is made using the lifetime fits to the "standard" and "EURODEC" $b$ baryon enriched Monte Carlo samples. The bias observed in the two samples are consistent, and the weighted average bias is, $(-7 \pm 2) \%$. Hence a correction of +0.07 ps is applied to the fit lifetime of the data sample.
Table 2

Summary of the systematic corrections to the lifetime result due to the various effects considered in the analysis. Recall that the statistical error in the result contains about 0.1 ps uncertainty owing to the fact that the background parametrisation is allowed to vary in the fit.

\begin{tabular}{lr}
\hline Source & Systematic (ps) \\
\hline decay length estimate bias & $+0.07 \pm 0.05$ \\
primary vertex position & $0.00 \pm 0.01$ \\
b baryon polarization & $0.03 \pm 0.03$ \\
b baryon momentum estimation & $0.00 \pm 0.05$ \\
b baryon mass & $+0.02 \pm 0.03$ \\
background charge invariance & $0.00 \pm 0.01$ \\
total & $+0.12 \pm 0.08$ \\
\hline
\end{tabular}

The reason for this bias is not completely understood. To investigate the effect, two other decay length estimators are considered to see if the full Monte Carlo sample correctly reproduces the differences between the different lifetime estimates observed in the data. The decay length estimator which uses the momentum sum of the $\Lambda$ and lepton (instead of the direction of the jet containing the lepton) as the directional constraint is seen to have a significant bias in the Monte Carlo samples. This comes about from a correlation between the orientation of the secondary vertex error ellipse and the directional constraint. The second decay length estimator considered is one which uses no directional constraint. In both cases the full Monte Carlo fit reproduces the differences in the lifetime estimates observed in the data within 0.05 ps. The systematic error assigned to the decay length estimate bias is therefore assigned to be $0.05 \mathrm{ps}$.

\subsection{Primary vertex position}

To determine the primary vertex, the LEP fillaveraged beam spots are used in addition to the tracks in the event. The lifetime analysis is quite insensitive to the estimated position and width of the beam spot. 
The size of the effect is estimated by observing the change in the lifetime estimate of the "standard" enriched $b$ baryon Monte Carlo sample when the beam position is shifted from its correct value by $20 \mu \mathrm{m}$ horizontally or vertically, or doubling the vertical beam size. The systematic uncertainty due to poorly defined beam spots and widths is found to be $0.01 \mathrm{ps}$.

A slight bias in the decay length estimator results from using the tracks in the event to help determine the primary vertex. If this was a large effect, it would be necessary to assign a systematic uncertainty in the modelling of this bias. The magnitude of this bias is determined with the "standard" enriched b baryon Monte Carlo sample by comparing the lifetimes determined with and without the aid of tracks in the event to define the primary vertex. The difference in the lifetimes is only $0.02 \mathrm{ps}$, and hence no additional systematic error is included.

\subsection{Estimation of the $b$ baryon momentum spectrum}

Since the b baryon momentum spectrum is estimated from the data, there is no systematic error resulting from the uncertainty in simulation of $b$ quark fragmentation. The method to estimate the $b$ baryon momentum spectrum, however, is sensitive to uncertainties in the modelling of the $b$ and $c$ baryon decays.

The $b$ baryons could be produced with large polarization in $\mathrm{Z}^{0}$ decays and this is not accounted for in the standard JETSET simulation of their decays. When the "polarization" Monte Carlo sample is used to define the $g(y)$ function the lifetime estimate increases by 0.06 ps. It is not known how much of the initial polarization is transferred to the $b$ baryons, and hence the range of polarizations from 0 to maximum is encompassed by applying a correction of $+0.03 \pm 0.03$ ps to the lifetime measurement.

In addition to polarization effects, the general simulation of the decay of $b$ and $c$ baryons has an uncertainty which can be treated as an uncertainty in the $g(y)$ function. The procedure for estimating the b baryon momentum spectrum results in mean values of 33.3 and $34.4 \mathrm{GeV} / c$ for the data and Monte Carlo, respectively. The difference between the two can arise from deficiencies in the modelling of $b$ and $c$ baryon decays and $b$ quark fragmentation. If the "EURODEC" Monte Carlo sample is used instead of the "standard" enriched Monte Carlo sample to define the $g(y)$ function, the reconstructed mean b baryon momentum for the data is $32.5 \mathrm{GeV}$. The effect of polarization, discussed above, could be contributing to the difference between the reconstructed $b$ baryon momenta of the data and Monte Carlo. Nevertheless, the systematic uncertainty of the mean $b$ baryon momentum estimation due to decay modelling alone is taken to be $2 \mathrm{GeV} / c$. This corresponds to a systematic uncertainty in the lifetime estimate of $0.05 \mathrm{ps}$.

\subsection{Mass of the $b$ baryon}

The $b$ baryon mass enters the model, when converting from decay times to decay lengths (eq. (3)). Although a nominal $\Lambda_{b}$ mass of $5.6 \mathrm{GeV} / c^{2}$ is used in the analysis, Monte Carlo studies indicate that many of the selected events could be from decays of $\Xi_{b}$, which is expected to have a mass about $0.2-0.3 \mathrm{GeV} / c^{2}$ greater. The $b$ baryon mass is therefore chosen to be $5.7 \pm 0.2 \mathrm{GeV} / c^{2}$. By repeating the analysis with different $b$ baryon masses, it is found that this introduces a correction of $+0.02 \pm 0.03$ ps to the measured lifetime.

\subsection{Background charge invariance}

In the analysis it is assumed that the background enters the right-sign and wrong-sign distributions with equal probability. With a subsample of the hadronic Monte Carlo data the backgrounds are found to equally populate the $\Lambda$-lepton combinations with the ratio of right-sign to wrong-sign background being $0.95 \pm 0.18$. By repeating the lifetime analysis of the data, this time scaling the background in the right-sign sample by $0.95 \pm 0.18$, the resulting lifetime estimate changes by at most 0.01 ps. The result is so insensitive to the scale of the background because the signal and background decay length distributions are very similar.

\section{Additional checks}

Checks for correlations between the lifetime estimate and all the event selection criteria give no evidence of a bias. This is done by breaking the event sample into pairs of subsamples and comparing the lifetime result in the two subsamples. This procedure 
Table 3

The results of miscellaneous checks of the stability of the lifetime analysis. The number of $\Lambda$-lepton combinations selected are shown as $n n n / m m m$ where $n n n$ is the number of right-sign and $m m m$ is the number of wrong-sign combinations.

\begin{tabular}{lll}
\hline Modification to standard analysis sample & Events & Lifetime estimate (ps) \\
\hline standard analysis & $261 / 103$ & $0.93_{-0.18}^{+0.20}$ \\
silicon information included & $250 / 101$ & $0.97_{-0.20}^{+0.23}$ \\
alternative $\Lambda$ selection \#1 & $156 / 72$ & $0.96_{-0.25}^{+0.29}$ \\
alternative $\Lambda$ selection \#2 & $250 / 113$ & $0.78_{-0.17}^{+0.21}$ \\
loose lepton selection & $420 / 223$ & $1.06_{-0.20}^{+0.21}$ \\
decay length window [-1,2] cm & $248 / 98$ & $0.98_{-0.19}^{+0.22}$ \\
and no secondary scale factors & $248 / 98$ & $0.98_{-0.18}^{+0.22}$ \\
\hline
\end{tabular}

also provides an additional check that the statistical error is properly evaluated.

Several checks that the result is stable under variations of the analysis are shown in table 3 . It is seen that the lifetime remains stable within 0.15 ps for all the changes to the analysis which are considered:

- The standard analysis, described above, is performed using track information which does not include information from the silicon vertex detector. When the analysis is repeated, this time using the silicon detector information, the result does not improve in precision because the decay length uncertainty is dominated by the $\Lambda$ track parameters (almost all of which decay beyond the radius of the silicon detector).

- Two alternative $\Lambda$ selections described in a recent paper [28] are used instead of the $\Lambda$ selection described above.

- The standard lepton selection is replaced with a much looser selection, resulting in a much larger background.

- The influence on the result from events with large decay lengths is checked by repeating the fit and excluding all events outside the decay length window $[-1,2] \mathrm{cm}$. Only a small change in the lifetime is observed. With this window cut, it is possible to fit the distribution without the secondary error scale factors (i.e. fix $f_{2}=0$ and $\tilde{f}_{2}=0$ in the fit). The result is the same as when all parameters are free.

\subsection{Check using the lepton impact parameters}

As a check, the b baryon lifetime is also determined using the impact parameters $\left(d_{0}\right)$ of the leptons mea- sured in the $r-\phi$ plane from the same data described above. The impact parameters are signed positive if the lepton track crosses the jet axis in the same hemisphere as the jet and negative otherwise. For signal events, the mean impact parameter is proportional to the $b$ baryon lifetime with the proportionality constant taken directly from all the $b$ baryon enriched Monte Carlo samples. To account for the background, the wrong-sign sample is used as follows:

$\tau=\frac{\tau_{\mathrm{MC}}}{\left\langle d_{0}\right\rangle_{\mathrm{MC}}} \frac{\left\langle d_{0}\right\rangle_{\mathrm{rs}}-f_{\mathrm{ws}}\left\langle d_{0}\right\rangle_{\mathrm{ws}}}{1-f_{\mathrm{ws}}}$,

where $\left\langle d_{0}\right\rangle_{M C}$ is the mean impact parameter of the b baryons in the Monte Carlo sample passing the selection criteria, $\tau_{M C}$ is the mean lifetime of these b baryons, $\left\langle d_{0}\right\rangle_{\mathrm{r}}$ and $\left\langle d_{0}\right\rangle_{\text {ws }}$ are the impact parameter means of the data right-sign and wrong-sign samples, respectively, and $f_{w s}$ is the ratio of the number of wrong-sign to right-sign events observed in the data.

In order to be less sensitive to the modelling of the detector resolution, a trimmed mean is used instead of the simple arithmetic mean. This involves removing an equal number of entries on each end of the distribution and then forming the average of the remaining entries. It is found in the Monte Carlo and data that this procedure gives a lifetime result relatively independent of the trim fraction, as long as more than about $20 \%$ trim is applied. For this trim value the b baryon lifetime is estimated to be $1.37 \pm 0.34$ ps.

A systematic error for this method is estimated by varying the trim amount between $12 \%$ and $70 \%$, considering the effects of jet axis resolution, and varying the Peterson fragmentation function parameter $\epsilon_{b}$ between 0.0025 and 0.0095 . Other systematic errors due 
to uncertainties in the background fraction, the primary vertex position, the $b$ baryon mass, and polarization are determined using similar methods as the main analysis. The combined systematic error from these effects is $0.13 \mathrm{ps}$. This method is more sensitive than the decay length method to errors in the modeling of $b$ baryon decays, but it is difficult to estimate the systematic uncertainty from this effect. Monte Carlo studies indicate the correlation coefficient between the decay length and impact parameter lifetime estimators is 0.7 . Hence the two results are consistent with each other even without considering the uncorrelated systematic uncertainties.

\section{Conclusions}

The average b-flavoured baryon lifetime is measured using the decay lengths of $\Lambda$-lepton vertices to be

$\tau=1.05_{-0.20}^{+0.23} \pm 0.08 \mathrm{ps}$,

where the first error is the statistical uncertainty and the second is the systematic. The statistical error in the result contains about 0.1 ps uncertainty owing to the fact that the background parametrisation is allowed to vary in the fit. The result agrees with the results $[3,6]$ already published by the ALEPH and DELPHI collaborations of $\tau=1.12_{-0.29}^{+0.32} \pm 0.16$ ps and $\tau=$ $1.04_{-0.38}^{+0.48} \pm 0.09 \mathrm{ps}$, respectively. These results can be compared to an average of recent $b$ hadron lifetime measurements [29] of $\tau=1.49 \pm 0.03$ ps.

\section{Acknowledgement}

It is a pleasure to thank the SL Division for the efficient operation of the LEP accelerator and their continuing close cooperation with our experimental group. In addition to the support staff at our own institutions we are pleased to acknowledge the Department of Energy, USA, National Science Foundation, USA, Texas National Research Laboratory Commission, USA, Science and Engineering Research Council, UK, Natural Sciences and Engineering Research Council,
Canada,

Fussefeld Foundation, Israeli Ministry of Energy and Ministry of Science, Minerva Gesellschaft, Japanese Ministry of Education, Science and Culture (the Monbusho) and a grant under the Monbusho International Science Research Program,

German Israeli Bi-national Science Foundation (GIF),

Direction des Sciences de la Matière du Commissariat à l'Energie Atomique, France,

Bundesministerium für Forschung und Technologie, Germany,

National Research Council of Canada,

A.P. Sloan Foundation and Junta Nacional de Investigação Científica e Tecnológica, Portugal.

\section{References}

[1] R.Rückl, in: Z Physics at LEP, CERN 89-08, Vol. 1 (1989) 311;

I.I. Bigi and N.G. Uraltsev, Phys. Lett. B 280 (1992) 271;

G. Altarelli and S. Petrarca, Phys. Lett. B 261 (1991) 303.

[2] ALEPH Collab., D. Buskulic et al., Phys. Lett. B 295 (1992) 174, 396;

DELPHI Collab., P. Abreu et al., Z. Phys. C 53 (1992)

567 ;

L3 Collab., B. Adeva et al., Phys. Lett. B 270 (1991)

111 ;

OPAL Collab., P.D. Acton et al., Measurement of the Average $b$ Hadron Lifetime in $Z^{0}$ Decays, CERNPPE/93-92, submitted to Z. Phys. (June 1993);

OPAL Collab., G. Alexander et al., Phys. Lett. B 266 (1991) 485.

[3] ALEPH Collab., D. Buskulic et al., Phys. Lett. B 297 (1992) 449.

[4] ALEPH Collab., D. Buskulic et al., Phys. Lett. B 307 (1993) 194.

[5] DELPHI Collab., P. Abreu et al., Phys. Lett. B 312 (1993) 253.

[6] DELPHI Collab., P. Abreu et al., Phys. Lett. B 311 (1993) 379.

[7] DELPHI Collab., P. Abreu et al., Z. Phys. C 57 (1993) 181.

[8] OPAL Collab., P.D. Acton et al., Phys. Lett. B 307 (1993) 247.

[9] OPAL Collab., P.D. Acton et al., Measurement of the $\mathbf{B}_{s}^{0}$ Lifetime, CERN-PPE/93-95, submitted to Phys. Lett. B (June 1993).

[10] H.-Y. Cheng, Phys. Lett. B 289 (1992) 455. 
[11] Particle Data Group, Review of Particle Properties, Phys. Rev. D 45 (1992) 1.

[12] OPAL Collab., K. Ahmet et al., Nucl. Inst. Meth. A 305 (1991) 275.

[13] M. Hauschild et al., Nucl. Inst. Meth. A 314 (1992) 74.

[14] OPAL Collab., G. Alexander et al., Z. Phys. C 52 (1991) 175.

[15] OPAL Collab., P.D. Acton et al., Phys. Lett. B 281 (1992) 394.

[16] OPAL Collab., P.D. Acton et al., Z. Phys. C 55 (1992) 191.

[17] OPAL Collab., P.D. Acton et al., Measurement of $\Gamma\left(\mathrm{Z}^{0} \rightarrow \mathrm{b} \overline{\mathrm{b}}\right) / \Gamma\left(\mathrm{Z}^{0} \rightarrow\right.$ hadrons $)$ using Leptons, CERN-PPE/93-46, submitted to Z. Phys. (March 1993).

[18] OPAL Collab., M.Z. Akrawy et al., Phys. Lett. B 263 (1991) 311.

[19] JADE Collab., W. Bartel et al., Z. Phys. C 33 (1986) 23 ;

JADE Collab., S. Bethke et al., Phys. Lett. B 213 (1988) 235.

[20] OPAL Collab., M.Z. Akrawy et al., Z. Phys. C 49 (1991) 375 .
[21] T. Sjöstrand, JETSET 7.3 Manual, CERNTH.6488/92;

OPAL optimised parameters were used, as described in OPAL Collab., M.Z. Akrawy et al., Z. Phys. C 47 (1990) 505.

[22] C. Peterson et al., Phys. Rev. D 27 (1983) 105.

[23] A. Ali and B. van Eijk, in: Z Physics at LEP, Vol. 3 (1989) 226.

[24] X.-H. Guo and P. Kroll, The $\Lambda_{b} \rightarrow \Lambda_{c}$ Decay Form Factors, WUB 92-07 (July 1992).

[25] T. Mannel and G. Schuler, Phys. Lett. B 279 (1992) 194;

T. Sjöstrand, private communication;

S. Jadach and $Z$. Was in $Z$ Physics at LEP, CERN 89-08, Vol. 1 (1989) 235.

[26] J. Allison et al., Nucl. Inst. Meth. A 317 (1992) 47.

[27] OPAL Collab., P.D. Acton et al., Measurement of the $\tau$ Lifetime, CERN-PPE/93-09, submitted to Z. Phys. (January 1993).

[28] OPAL Collab., P.D. Acton et al., Phys. Lett. B 291 (1992) 503.

[29] D. Karlen, B Hadron Lifetimes, Fifth International Symposium on Heavy Flavour Physics (Montréal, Canada, July 1993), to be published. 